\title{
Article
}

\section{Multi-Stage Meta-Learning for Few-Shot with Lie Group Network Constraint}

\author{
Fang Dong ${ }^{1,}$, Fanzhang $\mathrm{Li}^{1, *}$ \\ School of Computer Science and Technology, Soochow University, Suzhou 215006, Jiangsu, China; \\ 20185227041@stu.suda.edu.cn(F.D.) \\ * Correspondence: lfzh@suda.edu.cn
}

\begin{abstract}
Deep learning has achieved lots of successes in many fields, but when trainable sample are extremely limited, deep learning often under or overfitting to few samples. Meta-learning was proposed to solve difficulties in few-shot learning and fast adaptive areas. Meta-learner learns to remember some common knowledge by training on large scale tasks sampled from a certain data distribution to equip generalization when facing unseen new tasks. Due to the limitation of samples, most approaches only use shallow neural network to avoid overfitting and reduce the difficulty of training process, that causes the waste of many extra information when adapting to unseen tasks. Euclidean space-based gradient descent also make meta-learner's update inaccurate. These issues cause many meta-learning model hard to extract feature from samples and update network parameters. In this paper, we propose a novel method by using multi-stage joint training approach to post the bottleneck during adapting process. To accelerate adapt procedure, we also constraint network to Stiefel manifold, thus meta-learner could perform more stable gradient descent in limited steps. Experiment on mini-ImageNet[1] shows that our method reaches better accuracy under 5-way 1-shot and 5-way 5-shot conditions.
\end{abstract}

Keywords: meta-learning; lie group; machine learning; deep learning; convolutional neural network

\section{Introduction}

With the rapid improvement of computer performance and the scale of trainable data, deep learning models trained on large scale dataset could achieve huge improvement, some of them even has better performance than human in many scenes like classification[2] and regression. Most deep learning model could increase performance as the trainable data increase. But not every field has enough data using in training model, tradition deep learning models are difficult to have the same level learning ability and adaptability as humans when number of trainable samples is seriously insufficient.

One of the great advantages of human intelligence is that even children can learn to recognize new unseen things through few information. For example, give child a image of certain animal, and he can use it to grasp the feature of this species, and easily identify them when facing other images of this animal. The logic behind this example is that human beings can complete the construction of new task' $s$ feature and structures through the existing knowledge, and master faster and more accurate learning methods based on the continuous experience. Tradition deep learning models are dedicated to extracting embedding features from large scale data which belong to the same distribution and use them to classify new samples. Thus, once the trainable data amount is insufficient, or be required to quickly adapt to new unseen tasks, tradition models are difficult to deal with.

Meta-learning is used to explore and handle machine learning problems with limited trainable data. The state-of-the-art approach in this field is Model-Agnostic Meta-Learning[3], which denoted as MAML, this model provides a whole new structure to enhance the generalization performance. Generally, MAML-based models contain three parts: i) common initial basics; ii) adapting process and iii) gradient based updating rules to change network parameters inside meta-learner. These three parts correspond to the basic knowledge, learning process and learning approach in human's 
learning process in order. Meta-learning models usually embed a meta-learner which used to save and generate initial parameters. Meta-learner can adapt to new unseen tasks $\mathcal{T}$ by finetuning network parameters from few samples which has same classes in $\mathcal{T}$. After meta-learner adapting to unseen task $\mathcal{T}$, some new samples are used to measure the performance of meta-learner by accuracy, then model use optimizer to optimize the loss function object to update the whole model.

Since the adapting rule of meta-learner is fixed in MAML and model updates all parameters in meta-learner's network, MAML needs much more time to converge. Also, MAML starts training meta-learner from a randomly initialized network $\theta$, this force the model updates Feature Extractor and Classify Layer of meta-learner at the same time, Arnold et al.[4] point out that MAML-based models need much more network parameters than theoretically needed, because they should handle basic knowledge and new knowledge in one network, and meta-learner use low-level layers to extract features from sample data, use high-level layers such as full connect linear layer to classify.

As mentioned in Arnold's conclusion, tiny change in high-level layers contribute much more performance than layers in feature extractor during meta-test phase, so model can hardly optimize feature extractor and classify layer from few samples. Most of meta-learners in MAML-based methods only hold a shallow network which use few convolution layers as feature extractor. This factor further limits the meta-learner's ability to extract embedding features. In this paper, we tried deeper network structure by perform pretrain on meta-learner's feature extractor $\phi$. Many excellent methods[5][6][7] in meta-learning area execute pretrain to seek a feature extractor with enough generalization. Pretrained on large scale data could let meta-learner focus more on how to do better classify based on higher utilization of samples.

The updating rule is another issue of MAML that meta-learner perform evaluation on each step in adapting process, but only the last evaluation result been taken into to judge the performance of model. Meta-learner often executes multi updates in adapting process, it's obviously that test accuracy on new samples increase with the gradient descent performed on meta-learner's network and the increasement of accuracy is not linear related to steps, the increasement severely reduces in the middle and late stage in adapting process, updating process would easily falls into the bottleneck without manual intervention. Thus, we introduce multi-stage optimization method into MAML-based method. Model sets some checkpoints during the middle and late stage in adapting process to improve performance when stucks. This approach improves the utilization rate of training data under the limited update steps. Differential based gradient descent often applied in adapting process, therefore even if the network is very shallow, it still cannot guarantee the stability and the accuracy of direction in every gradient descent step, especially for the scene that trainable sample and number of update steps are extremely few.

In order to make up for the shortcomings that too much randomness in gradient descent, this paper introduces method which used in Manifold learning that define network under the orthonormal constraints. By constraint network geometry as Stiefel manifold or Lie group, the gradient on this network can be presented by Lie algebra and projection from orthogonal space $O(m)$ to Stiefel manifold space $S t(m, n)$. This constraint reduces the scale of parameter search space during gradient descent and improve the accuracy of gradient descent. In the remainder of this paper, we discuss detail of methods and then execute experiment on open source image dataset mini-ImageNet to evaluate our method.

The concept of meta-learning has been proposed decades ago, it first appear in education industry[8][9][10] to represent a frame that students should make better use of existing knowledge during learning and acquire better performance by mastering some basic knowledge and cooperating with continuous improvement of learning methods in the life-long learning process.

Many machine learning fields cannot prepare enough trainable data or models are required to adapt rapidly, meta-learning concept was introduced in machine learning. There are three main technical directions, first approach based on metric: Siamese Network[11] and Prototypical networks[12] calculate the similarity between samples, then classify them into predicted results by 
cosine distance; the second approach use a small extra neural network to replace gradient descent by outputting the corrected gradient value during adapting process[13] to makes meta-learner learn how to learn and what to learn rather than just learn a initial value; the third way based on gradient descent, most of them based on MAML's framework. These models update every layer of the neural network inside meta-learner by apply gradient descent. Specifically, MAML-based approach is the state-of-the-art algorithm in meta-learning field.

The detail of MAML algorithm is to embed meta-learner, a fully functional neural network model, into meta-learning model. It uses meta-learner to adapt to unseen tasks by learning from training data and output predictions. These methods constitute a dual-layer loop structure, this dual-layer loop allows model optimize the adapting process as a whole to equip initial knowledge with great generalization performance. MAML is also a framework for training and the design a model, thus it has better versatility even in reinforcement learning[14][15][16].

Based on MAML, Probabilistic model-agnostic meta-learning[17] introduces probabilistic into model to represent initial data, Kim[18] and Gupta[19] proposed Bayesian way into MAML to establish relationship between post result and pre data by Bayesian prior probability. Meta-SGD[20] and Task-Agnostic Meta-Learning(TAML)[21] try to make meta-learner learn how to learn better rather than only remember initial knowledge.

After deeper neural networks such as Residual network(ResNet)[22] and Dense network[23] been applied in machine learning, Qiao et al.[5]'s experiment shows that meta-learner could benefit from deeper and more complex network and model could has much better feature extractor ability, as mentioned by Arnold et al.[4] above. Training of meta-learner becomes harder with the scale expanded, which not only reflected in program running speed but also the stability during training.

To reduce the amount of parameters need to be updated, Rusu et al.[6] mapping parameters to a low-dimensional latent space as a way to reduce dimensions, then calculate gradients in latent space to make gradient descent more stable. Sun et al.[7] also work on this direction by reducing the number of parameters need to be optimized. We show that the most important part of MAML-based models is feature extractor in experiment. Our meta-test approach comes from Meta-Transfer Learning for Few-Shot Learning(MTL).

The idea that optimize the gradient descent itself by explore the geometry of homogeneous space exists for many years, often treat networks as matrices in Manifold learning[24][25] and Lie group learning[26][27] and constraint matrices to orthogonal group. Nishimori et al.[28] explore the gradient on high-dimension space and this allows meta-learner make better use of limited data and information in Euclidean space. Nishimori also derived this theory to a more general case which treat linear layer as Stiefel manifold which orthogonal constrainted and use Lie group transitive to simplify calculation process to a linear approximation. Yang et al.[29] has taken this approach in image classification problem and got excellent performance.

\section{Methods}

Meta-learning model using lots of tasks $\{\mathcal{T}\} \sim P(\mathcal{T})$ to train meta-learner which $\mathcal{T}$ is the smallest cell of training data, also denoted as meta-task. Training batch contains multi tasks, each task consists of $\left\{\mathcal{T}_{\text {tra }}, \mathcal{T}_{\text {qry }}\right\}$. Meta-learner updates network parameters by using $\mathcal{T}_{\text {tra }}$, then got prediction on $\mathcal{T}_{\text {qry }}$. The common setup for few-shot learning problem is (5-way 1-shot) and (5-way 5-shot), it means each task consists of 5 classes of data, the count of training samples is 1 or 5 , the number of samples used to update model denoted as Kshot. The diagram of $\mathcal{T}$ is shown in Figure 1.

For example, (5-way 1-shot) learning problem means meta-learner been used to classify 5 classes with only one trainable sample. A full train-test process contains two parts: meta - train and metatest, model training meta-learner in meta - train phase, then use datasets never been used in training process to test meta-learner's performance of adapting to new unseen tasks. 


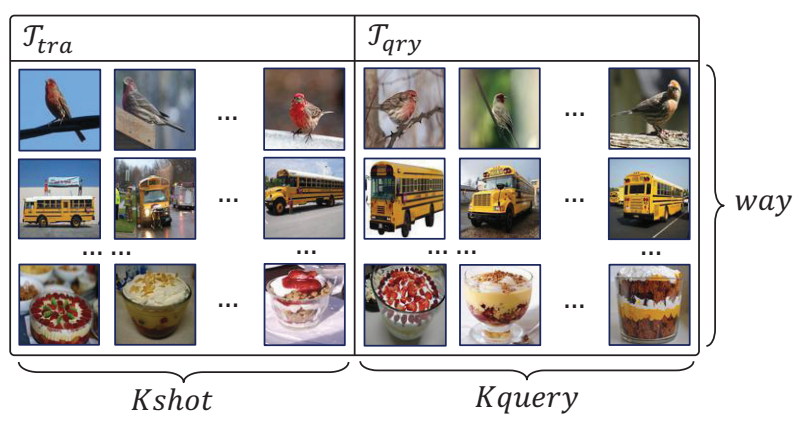

Figure 1. Overview of a task $\mathcal{T}$ used in meta-train phase includes $\mathcal{T}_{\text {tra }}$ and $\mathcal{T}_{\text {qry }}$. As described above, the number of $\mathcal{T}_{\text {tra }}$ is Kshot and the number of $\mathcal{T}_{\text {qry }}$ is Kquery.

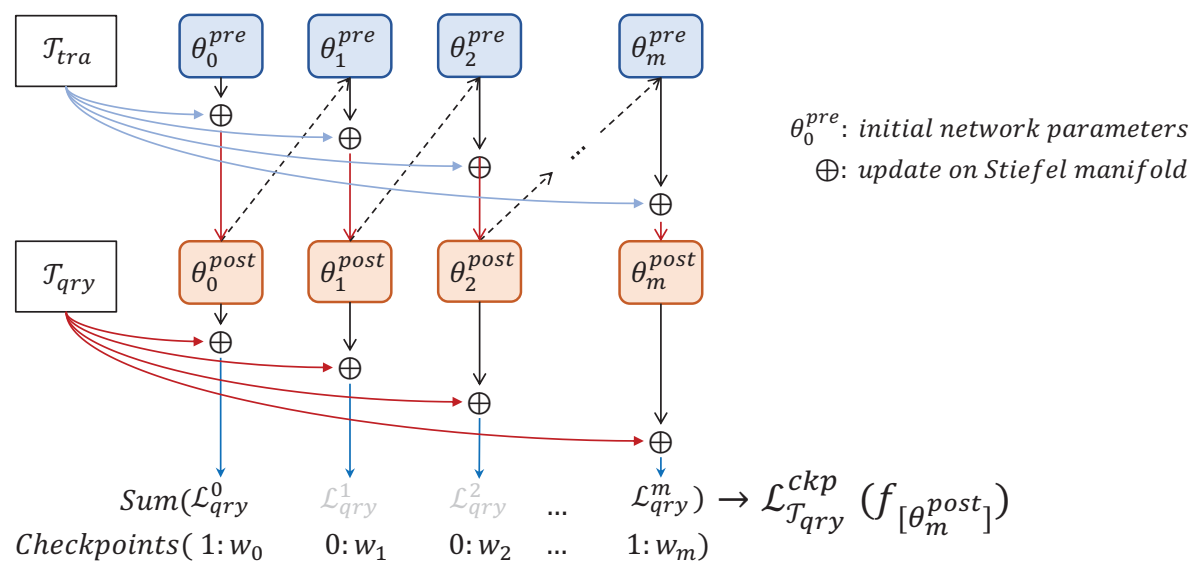

Figure 2. Diagram of our method, a fully inner loop process to optimize network $\theta$ in meta-learner.

Meta-task often splitted into two parts: $\left\{\mathcal{T}_{\text {tra }}, \mathcal{T}_{\text {qry }}\right\}$, there are Kshot samples in $\mathcal{T}_{\text {tra }}$ and Kqrery samples in $\mathcal{T}_{\text {qry }}$. When meta-learner finish learning from $\mathcal{T}_{\text {tra }}$, model generate loss function based on the prediction on $\mathcal{T}_{\text {qry }}$, then use optimizers such as SGD or Adam[30] to optimize it.

In this paper, we denote the process which meta-learner update its parameters by $\mathcal{T}_{\text {tra }}$ as inner loop, and denote the optimizing process on meta-learning model as outer loop. Therefore, we conclude that the largest different between no-meta-learning structure model and MAML-based model is that MAML-based model not only optimize the performance of meta-learner's prediction accuracy but also make it possible that optimize the process as a whole, no matter what meta-learner did and how it did during inner loop. After every inner loop finished, model got a loss function object, this setup allows model to use almost any approach to optimize the result from inner loop process. The updating rules of inner and outer loop in MAML as follows.

$$
\begin{gathered}
\theta_{k, i}=\theta_{k, i-1}-\alpha \nabla_{\theta_{k, i-1}} \mathcal{L}_{\mathcal{T}_{k}} f\left(\theta_{k, i-1}\right) \\
\theta \leftarrow \theta-\beta \nabla_{\theta}\left(\sum_{\mathcal{T}_{k} \sim \text { Batch }} \mathcal{L}_{\mathcal{T}_{k}}\left(f\left(\theta_{k, m}\right)\right)\right)
\end{gathered}
$$

\subsection{Pretrained by Large Scale Data}

Meta-learner needs large scale trainable data to equip generalization when facing unseen tasks, but its extreme unstable when use few samples to updating the whole network. Thus, MAML and many MAML-based models trend to use very shallow network which only contain 4 convolutional layers and a few full connect linear layers to avoid serious overfitting problem, but meta-learner requires high quality and accurately feature extraction from samples, as mentioned before, its almost impossible to update parameters of feature extractor $\phi$ and classify layer $\theta$ at the same moment. 
Training of parameters of meta-learner's network starting from randomly initialization in meta-train phase, it causes that every single parameter in meta-learner requires been updated to fit few-shot setup conditions. The whole model should focus more on how to adapt under a series of limitations rather than learns how to extract features from samples. Recently many meta-learning models split meta-learner into two pieces logically: Feature extractor and Classify layer, thus some models trend to pretrain the feature extractor part on large scale dataset[5][6][7]. Starting meta-train phase after pretrain process completed can greatly reduce the pressure of meta-learner.

Models in few-shot learning and some transfer learning approaches actually learn bias which related to new tasks of their parameters[31]. Our approach of pretrain consists with Sun et al. [5], first construct a model with deep neural network trained on mini-ImageNet dataset's subset [train $\cup$ val], then save weights except classify layer. Additionally, we transfer these parameters $\phi$ to another model to classify dataset's test set and only update last classify layer to confirm the performance of this feature extractor. updating rule shows in equation (3).

$$
\theta \leftarrow \theta-\alpha \nabla_{\theta_{f c}} \mathcal{L} f(\theta)
$$

We draw conclusion that it's possible to extract features from other data with different classes using network trained on large scale dataset. The pretrain algorithm is summarized in Algorithm 1.

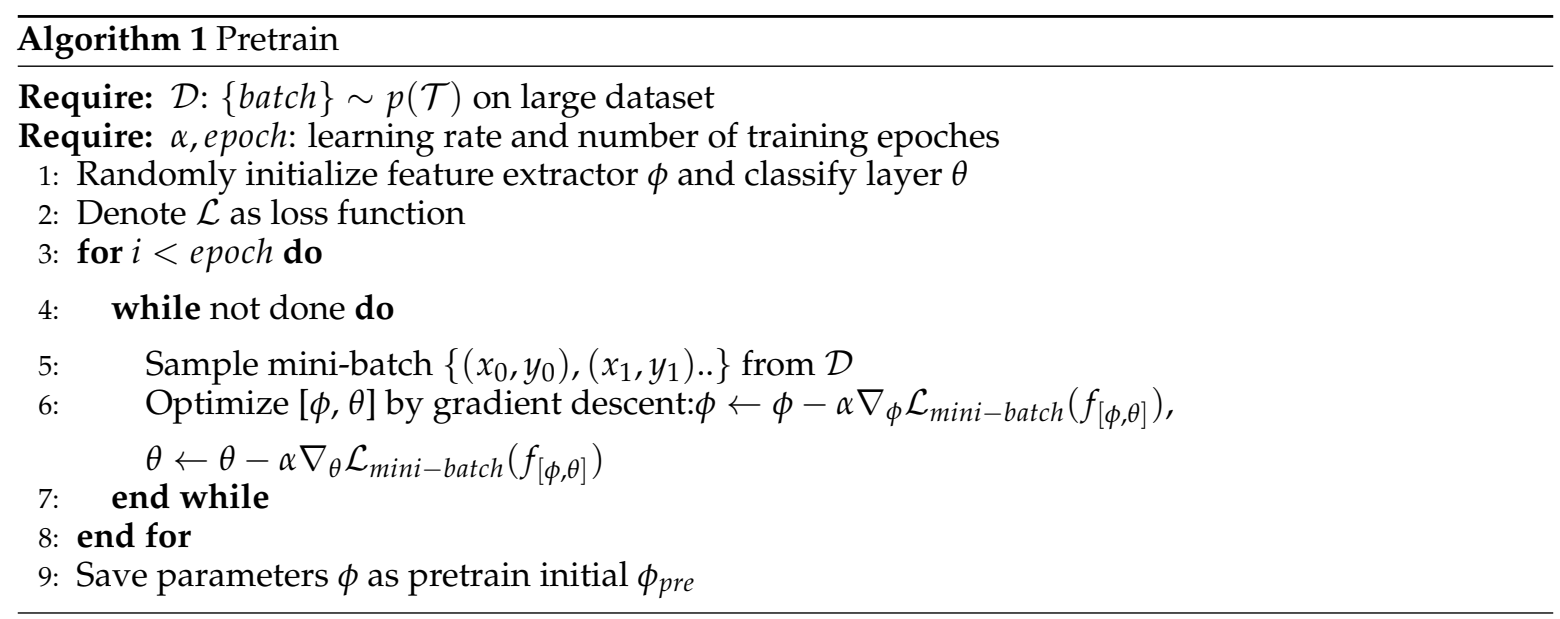

Training feature extractor on different dataset could reduce the burden of meta-learner during adapting processes and improve the whole model performance significantly. We modify updating methods both in inner loop and outer loop, for inner loop part, meta-learner only apply gradient descent on its classify layer and fix other parameters, this can speed up program speed and reduce space cost; for outer loop, still not every parameter get optimized, model optimizes meta-learner's classify layer, all bias, convolutions layers which its filter's kernel size is $(1 \times 1)$ and related batch normalization parameters. Specifically, our meta-learner uses wide residual network[32] with deep scale $W R N-K$ for 10 , selective optimization reduce the number of parameters need to be updated by $90.9 \%$ compared to method of MTL[7]. Our optimize principle trends to updating inductive biases of network to keep the ability of continuous adapting.

\subsection{Multi-Stage Optimization}

Meta-learning often compared to human's growth process, such as the initialization parameters learned through meta-train phase are similar to the structured general knowledge formed during the human learning process, the method used in inner loop could be seen as the way human learn new things. Inspired by pedagogy, a long education process can be splitted into several stages, human often 
test the performance at regular intervals to identify problems and make improvements on a timely basis rather than only focus on last exam result.

Inner loop process is similar to human education, the increasement of performance during inner loop adapting process are not linear, the accuracy on $\mathcal{T}_{\text {qry }}$ increase rapidly in first half but almost stopped increasing at the middle even if there are still steps to end. Other MAML-based approaches only focused on what meta-learner does in last step of inner loop[3][33][20] and they do not optimize the performance in time when meta-learner enter the bottleneck, it causes that lots of hidden information been wasted during adapting processes.

Under the premise of optimizing the result of the last step, we add some checkpoints to match inner loop's bottleneck places. This ensures that the accuracy keep increase to post the difficult points and keep the accuracy until meets the end. Meta-learner produces a copy $\left[\phi, \theta^{\prime}\right]$ of network parameters $[\phi, \theta]$ at every step in inner loop. Model use these parameters to make predictions on $\mathcal{T}_{\text {qry }}$ which compared to the grand true labels to generate loss function of this step. The count of inner loop denoted as $m$, we manually decide where to be optimized. Checkpoints are indexes which denote the place to joint current loss function into whole loss function which would be optimized in outer loop. Checkpoints are as follows.

$$
\text { ckp }:=\left[r_{1}, r_{2}, . . r_{i}\right] \quad i<m
$$

When step index in $c k p$, generate loss function $\mathcal{L}_{\mathcal{T}_{\text {qry }}}\left(f_{\left[\phi, \theta^{\prime}\right]}\right)$ by using query data $\mathcal{T}_{\text {qry }}$, model collected $m+1$ loss functions after inner loop completed: $\mathcal{L}_{\mathcal{T}_{\text {qry }}^{c k p}}^{c\left(\theta^{\prime}\right.}\left(f_{\left[\phi, \theta^{\prime}\right]}\right)=\sum \mathcal{L}_{\mathcal{T}_{\text {qry }}}^{c k p_{i}}\left(f_{\left[\phi, \theta^{\prime}\right]}\right)$, then adjust their importance by multiplying the weight coefficient $W_{n}=\left[w_{1}, w_{2}, \ldots w_{i}\right], w_{x} \sim[0,1]$. The update approach of outer loop shows in following.

$$
[\phi, \theta] \leftarrow[\phi, \theta]-\beta\left(\operatorname{grad}_{\left[\phi^{\prime}, \theta\right]} W_{m} \otimes \sum \mathcal{L}_{\mathcal{T}_{q r y}}^{c k p_{i}}\left(f_{\left[\phi, \theta^{\prime}\right]}\right)\right)
$$

\subsection{Lie Group Network Constrained}

As mentioned before, meta-learning are required use as few steps as possible when adapting to novel tasks to satisfy the requirements of rapid adaptation.

Most meta-learning methods apply update by gradient descent using $\nabla f(\theta)$, but it's difficult for meta-learner to generate gradient which can along nearest line when network holds tons of parameters. The sensitivity of Euclidean gradient descent $E-G D$ to the initial value of learning rate also makes these models violate the original intention of the design such like Adaptability and Robustness. Although there are proofs that Euclidean space based gradient descent offers better performance than some other adaptive optimizers[34], but the conditions of meta-learning limits the number of steps, this reduce the upper limit of gradient descent.

Adaptive optimizers such like Adam[30], AdaDelta[35] and SGD[36], they usually have faster convergence speed during model training phase using same number of steps. When the number of steps decreases further, the advantage over E-GD becomes tiny, this could be explained as adaptive optimizers usually change the length of each steps but not edit the direction of descent $V$. Model works on classify layer's parameters $\theta$ in inner loop process, the single full connect linear layer structure allows us define $\theta$ as a matrix $w \in R_{m \times n}$, which dimension is $m \times n$, elements in this is $x \in \mathbb{R}$, set $m>n$ to simplify the expression.

Thus the output pass through feature extractor and classify layer of meta-learner can be modeled as:

$$
\text { output }=\operatorname{softmax}\left(w^{T} x+w_{\text {bias }}\right)
$$


Updating rule by $E-G D$ to optimize the loss function formed by outputs and grand true labels as follows, $\alpha$ is the step size.

$$
w \leftarrow w-\alpha \nabla_{w} \mathcal{L}_{x} f(w)
$$

The network's parameter search space is $R_{m \times n}$ when using $E-G D$, so it's difficult for model with few updates to reach the convergence in time. There perhaps some latent geometric structures among neural networks[28] and this allows us treat the full connection layer as a manifold. Gradient descents on manifold have some advantages to $E$-GD: i) manifold limits by conditions, this significantly reduce the scale of parameter space; ii) the gradients via geodesic flows offer better accuracy to speed up convergence process. The geodesics is a distance minimizing curve between points in space with or without structures. The optimizing process of neural network can be seen as connecting the initial value position and stationary point of the loss function with the shortest path. Orthogonal constraint often be used when considering add conditions to parameter matrix $w$ :

$$
w \in S t(m, n)=\left\{w \in R_{m \times n} \mid w^{T} w=I_{n}\right\}
$$

Matrices satisfy this constraint are the points in Stiefel manifold, which can be seen as a Lie group, it means all $w$ of networks updating process constitute Stiefel manifold which belong to a $O(m, n)$ homogeneous space as the subspace of orthogonal Lie group $O(m)$. Denote $S t(m, n)$ as a Stiefel manifold with dimension $m \times n, O(m, n)$ is an orthogonal space with dimension $m \times n$ and $O(m)^{\prime}$ s dimension is $m \times m$. The regular way calculating geodesic on the manifold is searching nearest subset from start point to target position[37][38].

Firstly, generate the neighborhood subset $\cup_{s}=\left\{x_{i} \mid 0<i<l\right\}$ of start point $x_{s}$ and analyze this subset by propose an invariant scalar product $g_{i j}\left(x_{i}\right)$ under the automorphism at the identity element position, then calculate $g_{i j}\left(x_{i}\right)$ and use results into equation follows.

$$
d\left(x_{i}, x_{j}\right)=\sqrt{\sum_{n}^{i, j=1} g_{i j}\left(x_{i}\right)\left(x_{i i}-x_{j i}\right)\left(x_{i j}-x_{j j}\right)}
$$

Find every $x_{i}$ which less than threshold $d$ by calculating the Euclidean distance between $x_{i}$ and $x_{j}$ in set $\cup_{s}=\left\{x_{i} \mid 0<i<l\right\}$. Repeat this process till comes up geodesic from start point $x_{s}$ to end $x_{e}$.

But this approach costs too much computing resource because every point search paths passed by has its neighborhood, these neighborhoods contain some elements which also have their own neighborhoods, this defect becomes more and more serious as the dimension of parameter space increases. $S t(m, n)$ usually be embedded into a $O(m)$ matrix: $\tilde{W}=(w, I(m, m-n))$ by adding $O(n-p)$ orthogonal column vectors, $\tilde{W}$ is a special case of Stiefel manifold when $m=n$. So it's possible to use Lie algebra to simplified geodesic searching process on orthogonal group. Linear mapping based on matrix multiplication could naturally projection $O(m)$ to $S t(m, n)$. Stiefel manifold itself is a homogeneous space, thus there is transitive action by Lie group on it. From initial point $\tilde{W}_{0}$, network value can reach every position in Stiefel manifold by left multiplication G-action $\tilde{W}_{1}=R \tilde{W}_{0}$ [26].

Denote $G(\tilde{W})=\{W \in S t(m, n) \mid W=R W, R \in O(m)\}$ as the space $R \tilde{W}_{0}$ could reached, it's equivalent to the parameter space with orthogonal constraint. So the method which update parameter matrix by multiplication a $O(m)$ is feasible. Nature gradient via geodesic flow on $O(m)$ can be obtained by Lie algebra exponential map, which is proposed by Yasunori[28]. 


$$
\begin{gathered}
\tilde{W} \leftarrow \tilde{W} \exp \left(-\gamma \tilde{W}_{n}^{t} \tilde{V}_{H}\right) \\
\tilde{V}_{H}=\lambda\left\{\nabla f(g(\tilde{W}))-\tilde{W} \nabla f(g(\tilde{W}))^{T} \tilde{W}\right\} \\
g(\tilde{W}): O(m) \rightarrow S t(m, n), \tilde{W} \in O(p), g(\tilde{W})=w \in S t(m, n)
\end{gathered}
$$

Linear approximation could avoid exponential calculations by projection gradient from $O(m)$ in $\tilde{W}$ to $S t(m, n)$ [39]. Natural gradient on Stiefel manifold described by the following formula.

$$
\operatorname{grad}_{S t}(f(w))=\nabla f(w)-w \nabla f(w)^{T} w
$$

Our method replaces the differential in Euclidean space with natural gradient in Stiefel manifold and comes up the updating rule in inner loop process.

$$
\theta^{\prime}=\theta-\alpha\left(\nabla_{\theta} \mathcal{L}_{\mathcal{T}_{\text {sup }}}\left(f_{[\phi, \theta]}\right)-\theta \times \nabla_{\theta}^{T} \mathcal{L}_{\mathcal{T}_{\text {sup }}}\left(f_{[\phi, \theta]}\right) \times \theta\right)
$$

We also compared the performance of the model using Stiefel manifold structure and E-GD under the same other conditions. The method that use linear approximation natural gradient descent guarantees the computational efficiency in backpropagation, and does not introduce new extra parameters. Approaches in meta-train phase presented in Algorithm 2.
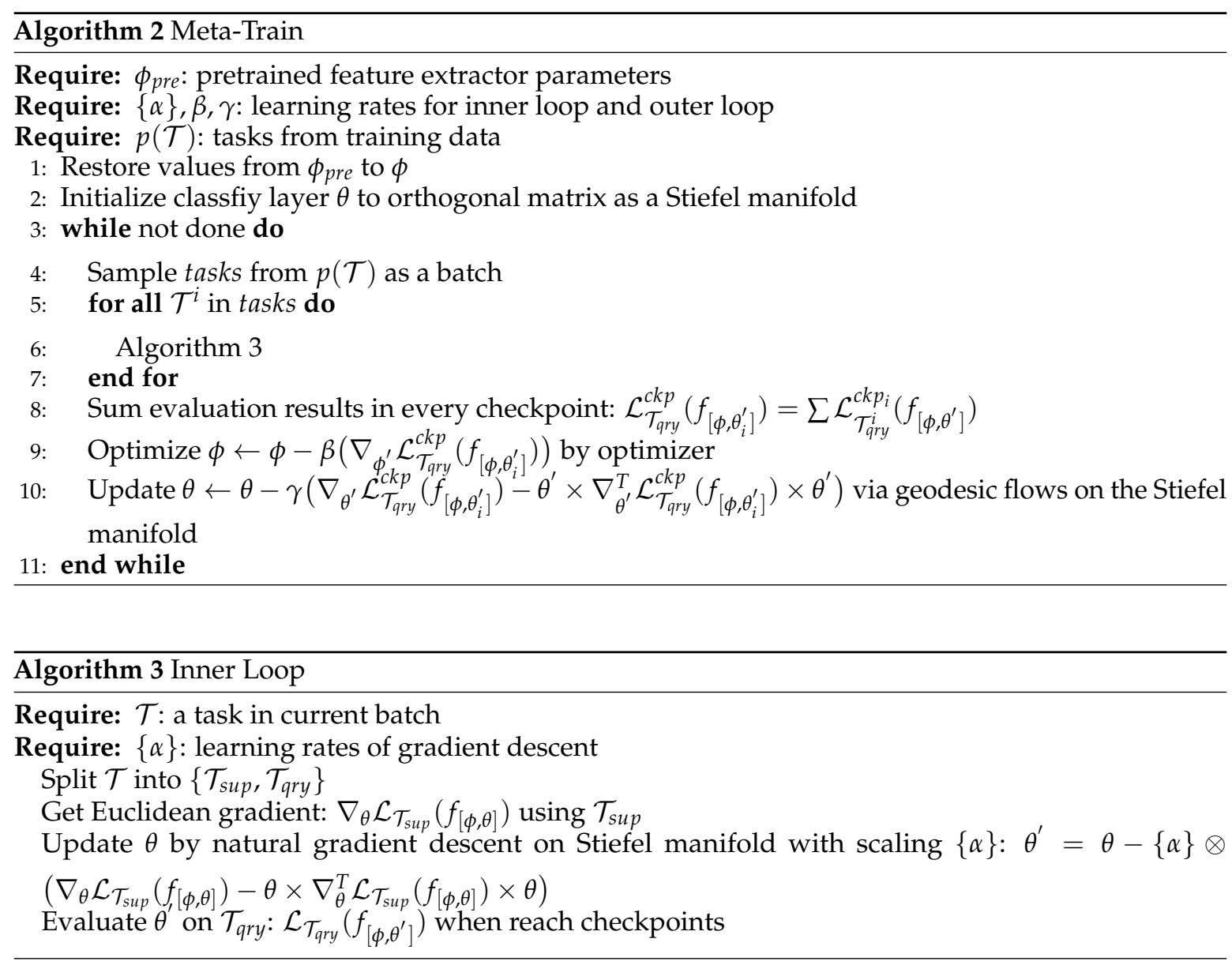


\section{Experiments}

\subsection{Dataset}

Dataset used in experiment is the universal image dataset used in few-shot learning area: mini-ImageNet[1], which is the subset of ImageNet[40]. Images in this dataset are natural photos, there are 100 classes pictures and each class contain 600 images which usually cropped to $80 \times 80$ pixels in deep learning models. Mini-ImageNet often be splitted into three parts: Train, Validation and Test. Training subset contains 64 classes images with 38400 samples, validation subset has 16 classes, 20 classes for test set.

\subsection{Model Detail}

Our completed training process consists of three phases: pretrain phase, meta-train phase and meta-test phase. Training set and val set were used during pretrain phase, pretrain model and meta-train model share the same network structure.

\subsubsection{Pretrain Phase}

We train a model on subsets [train $\cup$ val] of mini-ImageNet to classify 80 classes images. Network structure used in feature extractor part is wide residual network[32] with 22 convolutional layers, extended from Residual Network[22], the depth scale of filters in convolutional kernel is 10. Denote this network as WRN-22-10.

Input data preprocessed by the initial part Conv-Batch normalization-ReLU in model. Depth of residual block sets progression in three stages $[160,320,640]$, each residual block set has 3 residual blocks. Convolutional layer with $3 \times 3$ kernel size shrinks the feature map size with stride 2 and extend the depth, then Batch normalization and activation function ReLU[41] applied before data meet second convolution layer. After two $3 \times 3$ convolutional operations, the processed feature map been added to the original input data.

Specifically, we insert dropout layer between convolutional layers in each residual block to make its structure wide-dropout, the convolutional layers type we use is $B(3,3)$. According to Zagoruyko et al.[32], dropout layer inside blocks could prevent overfitting, the keep - rate of these dropout set at 0.5. Global average pooling with kernel size $=10$ applied before data reach the classify layer.

We use this WRN-22-10 as feature extractor and add 2 linear layers with ReLU, output nodes of classify layer is $[1000,5]$. Initial learning rate set in 0.1 which used in the SGD optimizer. Batch size is the smallest exponent of 2 greater than classes 80 at 128. Fully pretrain phase contains 100 epochs, learning rate decay by $20 \%$ per 30 epochs.

\subsubsection{Meta Train Phase}

Because the gradient descent approach was changed to using natural gradient via geodesic, we use single linear layer as the classify layer. The structure of feature extractor in meta-train phase was same as pretrain model, whom parameters extended from pretrained model. Wide-drop[32] also been used in meta-train stage but disabled in meta-test phase.

Parameters splitted into two parts during meta-train: classify layer $\theta$ and biases of feature extractor $\phi^{\prime}$. Initial learning rate of inner loop use decay strategy, the learning rate multiply 0.99 after every gradient descent. Loss function generated by query data $\mathcal{T}_{\text {qry }}$ and $\left[\theta^{\prime}, \phi\right]$ was optimized in outer loop. Optimizer used on parameters in feature extractor is Adam[30] with initial learning rate $1 e-4$. Network in classify layer was updated by Stiefel manifold natural gradient to keep matrix always orthogonal, the learning rate in this optimizing process is 0.01 .

Due to the feature extractor has been trained in pretrain phase, model's pressure in meta-train reduced, and the requirement of data decreased than MAML. The number of tasks using in meta-train 
phase is $5 \mathrm{~K}$ compared with $240 \mathrm{~K}$ used in MAML as batch size $=1$, the number of iterations is also $15 \mathrm{~K}$. Model uses weights trained on $[$ train $\cup$ val $]$ to test performance on test subset.

\section{Results}
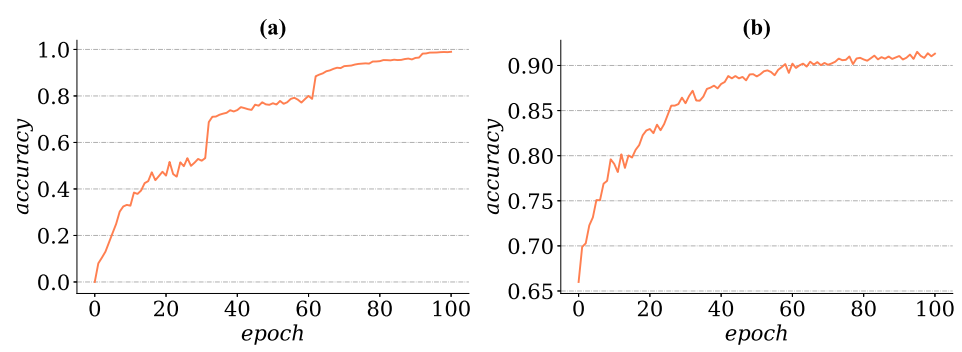

Figure 3. (a) Testing accuracy on mini-ImageNet dataset during pretrain phases for 100 epochs. (b) The accuracy that transfer feature extractor to classify test subset, result shows that model can reach the pretty high accuracy even only trains the classify layer.

(a)

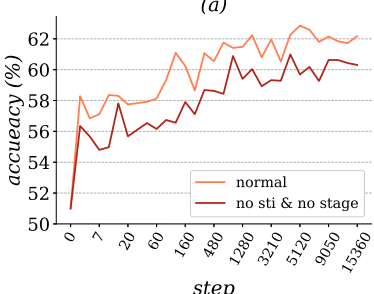

step (b)

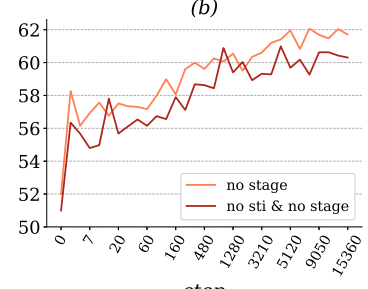

step (c)

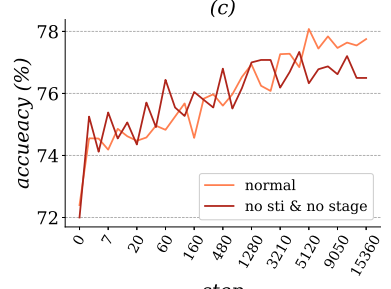

(d)

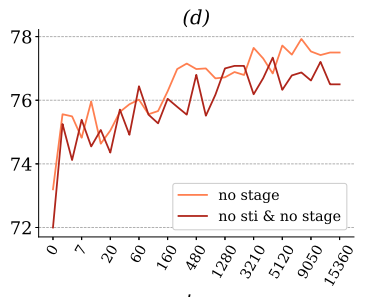

step

Figure 4. (a)(b) represent the accuracy results of 5-way 1-shot learning on mini-ImageNet dataset with different settings, no sti in figures means gradient descent is Euclidean gradient, no stage is the setting that disable the multi-stage optimizing method. (c)(d) show results under the setting 5-way 5-shot.

The classification accuracies of our method and other baselines are shown in Table 1. We achieved the state-of-the-art results of the 5-way 1-shot and 5-way 5-shot on mini-ImageNet dataset. Performance of 5-way 1-shot is similar to LEO[6]. Compared with our base model MTL[7], our method proves a certain improvement by using manifold learning approach.

We also present contributions from each method we proposed, results show that deeper network provide significant improvements on feature extraction. The application of Stiefel manifold learning makes the performance of model increase by almost $1 \%$ on the basis in 5-way 1-shot learning problem. It's clear that feature extraction performance reaches the bottleneck when transfer to test set, multi-stage optimization provide tiny improvements to the model. In the setting of machine learning, especially for deep learning, the data in a dataset belong to a certain distribution, which make it possible for neural network to learn the common information from the dataset, but whether these data actually belong to a latent distribution is still unknown, so in a dataset there is a gap on training feature extraction capabilities on subset 1 and applying it to subset 2 .

\section{Discussion}

We introduced three approaches to solve problems of MAML: i) feature extraction; ii) information utilization and iii) the efficiency of gradient descent. By saving intermediate states during adapting processes, we postponed the position of bottleneck to increase the performance of model. Network constrainted to Stiefel manifold offers better descent accuracy. These methods make our model demonstrated the new state-of-the-art result under the setting of 5-way 1-shot and 5-shot.

But our model still cannot automatically improve existing feature extractor when encountering new datasets, this limits the model's upper limit of performance even trained and tested on a same dataset. Because the optimization process of the model is logically divided into two parts, and they 
Table 1. Image classification results on mini-ImageNet under the setting of 5-way 1-shot or 5-way 5-shot. Results in this table reported from their papers.

\begin{tabular}{lcc}
\hline Few-shot learning method & 1-shot & 5-shot \\
\hline Metric Based & & \\
Matching Nets[1] & $43.56 \pm 0.84 \%$ & $55.31 \pm 0.73 \%$ \\
Relation Nets[42] & $50.44 \pm 0.82 \%$ & $65.32 \pm 0.70 \%$ \\
Prototypical Nets[12] & $49.42 \pm 0.78 \%$ & $68.20 \pm 0.66 \%$ \\
\hline Gradient descent based & & \\
MAML[3] & $48.70 \pm 1.84 \%$ & $63.11 \pm 0.92 \%$ \\
Reptile[33] & $49.97 \pm 0.32 \%$ & $65.99 \pm 0.58 \%$ \\
meta-SGD[20] & $50.47 \pm 1.87 \%$ & $64.03 \pm 0.94 \%$ \\
LEO[6] & $61.76 \pm 0.15 \%$ & $77.46 \pm 0.12 \%$ \\
MTL[7] & $61.20 \pm 1.80 \%$ & $75.50 \pm 0.80 \%$ \\
TAML[21] & $51.73 \pm 1.88 \%$ & $66.05 \pm 0.85 \%$ \\
\hline Memory augmented based & & \\
TADAM[43] & $58.50 \pm 0.30 \%$ & $76.70 \pm 0.30 \%$ \\
SNAIL[44] & $55.71 \pm 0.99 \%$ & $68.66 \pm 0.92 \%$ \\
\hline MSML(Ours) No Sti & $\mathbf{6 1 . 1 2} \pm \mathbf{1 . 0 2} \%$ & $\mathbf{7 5 . 1 3} \pm \mathbf{0 . 4 1} \%$ \\
MSML(Ours) No Stages & $\mathbf{6 1 . 5 0} \pm \mathbf{0 . 8 2} \%$ & $\mathbf{7 7 . 2 1} \pm \mathbf{0 . 7 3} \%$ \\
MSML(Ours) & $\mathbf{6 2 . 4 2} \pm \mathbf{0 . 7 6} \%$ & $\mathbf{7 7 . 3 2} \pm \mathbf{0 . 6 6} \%$ \\
\hline
\end{tabular}

use completely different calculation methods and corresponding learning rates, how to coordinate the step size between the two parts of the model through the adaptive learning rate is also a question to solved. These are works need to be studied in the future to improve the adaptability of model.

\section{Materials and Methods}

\subsection{Computational Requirements}

Code used in experiments based on PyTorch and runs on Linux. Softwares required are Python 3.6, CUDA and cuDNN. We recorded the resource usage when running these experiments, program requires 10.1GB GPU memory and 1.8GB memory under the setup of 5-way 5-shot learning.

\subsection{Program Availability}

Dataset and runnable code used in our experiments are available in https:/ / github.com/Chacrk/ MSML_Project, detailed running instructions is also available in repository.

\subsection{Running Experiment}

Download mini-ImageNet dataset, images are croped to $(84 \times 84)$, unzip file by run command python3 proc_dataset.py in data folder. Enter directory pretrain and run python3 pretrain.py to get pretrained weights data.

Pretrain phase last about 5.1 hours by using NVIDIA TITAN Xp and Intel Xeon E5-2620 v4. Enter meta folder while pretrain process done and run command python3 main.py to run the experiment.

Author Contributions: All authors contributed to this article, conceptualization, F.D. and F.L.; software, F.D; validation, F.D. and F.L.; writing-original draft preparation, F.D; writing-review and editing, F.D. and F.L.; visualization, F.D.; supervision, F.L.; funding acquisition, F.L.

Funding: This research is supported by National Natural Science Foundation of China under grant number 61902269 and National Key Research and Development Plan "Key Scientific Issues of Transformative Technology" of China(grant number 2018YFA0701701).

Acknowledgments: We would like to thank Yang Liu, Xiaohang Pan, Qi Cai, Zhe Wang, Pengxiang Wu and my tutor Fanzhang Li for technical support. Also we appreciated the research funds mentioned above and computers resources provided by Machine Learning Lab in Soochow University. 
Conflicts of Interest: The authors declare no conflict of interest.

\section{References}

1. Vinyals, O.; Blundell, C.; Lillicrap, T.; Wierstra, D.; others. Matching networks for one shot learning. Advances in neural information processing systems, 2016, pp. 3630-3638.

2. Szegedy, C.; Vanhoucke, V.; Ioffe, S.; Shlens, J.; Wojna, Z. Rethinking the inception architecture for computer vision. Proceedings of the IEEE conference on computer vision and pattern recognition, 2016, pp. 2818-2826.

3. Finn, C.; Abbeel, P.; Levine, S. Model-agnostic meta-learning for fast adaptation of deep networks. Proceedings of the 34th International Conference on Machine Learning-Volume 70. JMLR. org, 2017, pp. 1126-1135.

4. Arnold, S.M.; Iqbal, S.; Sha, F. Decoupling Adaptation from Modeling with Meta-Optimizers for Meta Learning. arXiv preprint arXiv:1910.13603 2019.

5. Qiao, S.; Liu, C.; Shen, W.; Yuille, A.L. Few-shot image recognition by predicting parameters from activations. Proceedings of the IEEE Conference on Computer Vision and Pattern Recognition, 2018, pp. 7229-7238.

6. Rusu, A.A.; Rao, D.; Sygnowski, J.; Vinyals, O.; Pascanu, R.; Osindero, S.; Hadsell, R. Meta-learning with latent embedding optimization. arXiv preprint arXiv:1807.05960 2018.

7. Sun, Q.; Liu, Y.; Chua, T.S.; Schiele, B. Meta-transfer learning for few-shot learning. Proceedings of the IEEE Conference on Computer Vision and Pattern Recognition, 2019, pp. 403-412.

8. Glass, G.V. Primary, secondary, and meta-analysis of research. Educational researcher 1976, 5, 3-8.

9. Powell, G. A Meta-Analysis of the Effects of" Imposed" and" Induced" Imagery Upon Word Recall. 1980.

10. Adey, P.; Shayer, M. Strategies for meta-learning in physics. Physics education 1988, 23, 97.

11. Koch, G.; Zemel, R.; Salakhutdinov, R. Siamese neural networks for one-shot image recognition. ICML deep learning workshop, 2015, Vol. 2.

12. Snell, J.; Swersky, K.; Zemel, R. Prototypical networks for few-shot learning. Advances in Neural Information Processing Systems, 2017, pp. 4077-4087.

13. Ravi, S.; Larochelle, H. Optimization as a model for few-shot learning 2016.

14. Gupta, A.; Eysenbach, B.; Finn, C.; Levine, S. Unsupervised meta-learning for reinforcement learning. arXiv preprint arXiv:1806.04640 2018.

15. Nagabandi, A.; Clavera, I.; Liu, S.; Fearing, R.S.; Abbeel, P.; Levine, S.; Finn, C. Learning to adapt in dynamic, real-world environments through meta-reinforcement learning. arXiv preprint arXiv:1803.11347 2018.

16. Al-Shedivat, M.; Bansal, T.; Burda, Y.; Sutskever, I.; Mordatch, I.; Abbeel, P. Continuous adaptation via meta-learning in nonstationary and competitive environments. arXiv preprint arXiv:1710.03641 2017.

17. Finn, C.; Xu, K.; Levine, S. Probabilistic model-agnostic meta-learning. Advances in Neural Information Processing Systems, 2018, pp. 9516-9527.

18. Kim, T.; Yoon, J.; Dia, O.; Kim, S.; Bengio, Y.; Ahn, S. Bayesian model-agnostic meta-learning. arXiv preprint arXiv:1806.03836 2018.

19. Yoon, J.; Kim, T.; Dia, O.; Kim, S.; Bengio, Y.; Ahn, S. Bayesian model-agnostic meta-learning. Advances in Neural Information Processing Systems, 2018, pp. 7332-7342.

20. Li, Z.; Zhou, F.; Chen, F.; Li, H. Meta-SGD: Learning to learn quickly for few-shot learning. arXiv preprint arXiv:1707.09835 2017.

21. Jamal, M.A.; Qi, G.J. Task Agnostic Meta-Learning for Few-Shot Learning. Proceedings of the IEEE Conference on Computer Vision and Pattern Recognition, 2019, pp. 11719-11727.

22. He, K.; Zhang, X.; Ren, S.; Sun, J. Deep residual learning for image recognition. Proceedings of the IEEE conference on computer vision and pattern recognition, 2016, pp. 770-778.

23. Zhang, Y.; Tian, Y.; Kong, Y.; Zhong, B.; Fu, Y. Residual dense network for image super-resolution. Proceedings of the IEEE Conference on Computer Vision and Pattern Recognition, 2018, pp. 2472-2481.

24. Cai, D.; He, X.; Han, J.; Zhang, H.J. Orthogonal laplacianfaces for face recognition. IEEE transactions on image processing 2006, 15, 3608-3614. 
25. Zhang, Z.; Chow, T.W.; Zhao, M. M-Isomap: Orthogonal constrained marginal isomap for nonlinear dimensionality reduction. IEEE transactions on cybernetics $2012,43,180-191$.

26. Fan-Zhang, L.; Huan, X. SO (3) classifier of Lie group machine learning 2007.

27. Li, F.z.; Xu, H. SO (3) classifier of Lie group machine learning. Learning 2007, 9, 12.

28. Nishimori, Y. A Neural Stiefel Learning based on Geodesics Revisited. WSEAS Trans. Syst. 2004, 3, 513-520.

29. Yang, M.; Li, F.; Zhang, L.; Zhang, Z. Lie group impression for deep learning. Information Processing Letters 2018, 136, 12-16.

30. Kingma, D.P.; Ba, J. Adam: A method for stochastic optimization. arXiv preprint arXiv:1412.6980 2014.

31. Battaglia, P.W.; Hamrick, J.B.; Bapst, V.; Sanchez-Gonzalez, A.; Zambaldi, V.; Malinowski, M.; Tacchetti, A.; Raposo, D.; Santoro, A.; Faulkner, R.; others. Relational inductive biases, deep learning, and graph networks. arXiv preprint arXiv:1806.01261 2018.

32. Zagoruyko, S.; Komodakis, N. Wide residual networks. arXiv preprint arXiv:1605.07146 2016.

33. Nichol, A.; Achiam, J.; Schulman, J. On first-order meta-learning algorithms. arXiv preprint arXiv:1803.02999 2018.

34. Keskar, N.S.; Socher, R. Improving generalization performance by switching from adam to sgd. arXiv preprint arXiv:1712.07628 2017.

35. Zeiler, M.D. ADADELTA: an adaptive learning rate method. arXiv preprint arXiv:1212.5701 2012.

36. Bottou, L. Large-scale machine learning with stochastic gradient descent. In Proceedings of COMPSTAT'2010; Springer, 2010; pp. 177-186.

37. Belkin, M.; Niyogi, P. Semi-supervised learning on Riemannian manifolds. Machine learning 2004, 56, 209-239.

38. Li, F. Lie Group Machine Learning; Press of University of Science and Technology of China, 2013.

39. Nishimori, Y.; Akaho, S. Learning algorithms utilizing quasi-geodesic flows on the Stiefel manifold. Neurocomputing 2005, 67, 106-135.

40. Deng, J.; Dong, W.; Socher, R.; Li, L.J.; Li, K.; Fei-Fei, L. Imagenet: A large-scale hierarchical image database. 2009 IEEE conference on computer vision and pattern recognition. Ieee, 2009, pp. 248-255.

41. Maas, A.L.; Hannun, A.Y.; Ng, A.Y. Rectifier nonlinearities improve neural network acoustic models. Proc. icml, 2013, Vol. 30, p. 3.

42. Sung, F.; Yang, Y.; Zhang, L.; Xiang, T.; Torr, P.H.; Hospedales, T.M. Learning to compare: Relation network for few-shot learning. Proceedings of the IEEE Conference on Computer Vision and Pattern Recognition, 2018, pp. 1199-1208.

43. Oreshkin, B.; López, P.R.; Lacoste, A. Tadam: Task dependent adaptive metric for improved few-shot learning. Advances in Neural Information Processing Systems, 2018, pp. 721-731.

44. Mishra, N.; Rohaninejad, M.; Chen, X.; Abbeel, P. A simple neural attentive meta-learner. arXiv preprint arXiv:1707.03141 2017. 